Research Article

\title{
Large Amplitude Free Vibrations of Mindlin Square Plates using Coupled Displacement Field Method
}

\author{
K.Meera Saheb ${ }^{\dot{A}^{*}}$ and M.Shasikanth ${ }^{\dot{B}}$ \\ ${ }^{\grave{A}}$ Department of Mechanical Engineering University college of engineering(A), JNTU Kakinada India \\ ${ }_{\mathrm{B}}^{\mathrm{B}}$ Department of Mechanical Engineering, Malla Reddy College of Engineering, Hyderabad, India \\ Accepted 10 January 2014, Available online 01 February 2014, Special Issue-2, (February 2014)
}

\begin{abstract}
A simple and efficient coupled displacement field method is developed to study the large amplitude free vibration behavior of the moderately thick square plates. A single term trigonometric admissible displacement field is assumed for one of the variables, say, and the total rotations (in both $X, Y$ directions). Making use of the coupling equation, the spatial variation for the lateral displacement field is derived in terms of the total rotations. The coupled displacement field method makes the energy formulation to contain half the number of unknown independent coefficients, in the case of a square plate, contrary to the conventional Rayleigh-Ritz method. The lesser number of undetermined coefficients significantly simplifies the vibration problem. The expressions for the linear and nonlinear fundamental frequency parameters for the all edges simply supported and clamped moderately thick square plates are derived. The numerical results obtained from the present formulation are in very good agreement with those obtained from the existing literature.
\end{abstract}

Keywords: The Mindlin square plates, Large amplitude free vibrations, CDFMmethod

\section{Introduction}

Knowledge of fundamental frequency parameters of moderately thick plates is important and has to be considered in the initial design phase of such structural members. The energy methods provide a convenient means for computing the fundamental frequency parameters of these structural members and the solutions obtained using this approach are upper bounds and the accuracy of the solution depends on the admissible functions chosen for the lateral displacement and total rotations.

The concept of coupled displacement field (CDF) which was successfully used in the finite element(FE) analysis and reported in the open literature. A continuum analogy of the FE analysis with the CDF method has not received much attention except in the formulation of Zhou (2001) where the two fields (displacement and rotation) are coupled through an equation which is dependent on the applied load and is different from the FE method. The concept of coupled displacement field method to study the large amplitude free vibration behavior of uniform Timoshenko beams has been demonstrated successfully (G.Venkateshwar Rao et al 2004)( G.Venkateshwar Rao et al 2005)(K Meera Saheb 2005 et al). The energy method gives exact solutions if the chosen admissible functions represent the mode shape of vibration exactly. Singh and Rao et al (1978) have studied the large amplitude free

*Corresponding author: K.Meera Saheb

DOI: http://dx.doi.org/10.14741/ijcet/spl.2.2014.129 vibration of isotropic and composite moderately thick square plates using the FE formulation. In this study the authors made an effort to study the free vibration of uniform shear deformable moderately thick square plates using the coupled displacement field method.

The fundamental frequency parameters for the thin square plates are evaluated in Refs. (H.N Chu et al 1956)(Chu Mei 1973)(C.M Wang et al 2005) for several configurations of the plates including the different in plane edge load conditions and boundary conditions. If the plates are moderately thick, the effect of the shear deformation and rotary inertia have to be considered and this effect is introduced by another set of admissible function for the total rotation. Thus if $\mathrm{n}$ admissible functions are chosen for the total rotation, another $n$ admissible functions for the lateral displacement result in. This makes the vibration problems to contain $2 \mathrm{n}$ unknowns, in general, and the use of the classical R-R method(K.Meerasaheb et al 2009) yields 2 n homogeneous simultaneous equations which are to be solved for obtaining the fundamental frequency parameters. However, if an accurate single term admissible function is used in the R-R method, a two unknown coefficients problem has to be solved, where one unknown coefficient is associated with the lateral displacement / total rotation and the other unknown coefficient is associated with the total rotation / lateral displacement.

In this paper, the authors propose to use a new method called the CDF method and this significantly simplifies 
the formulation of the vibration problem of moderately thick square plates. In this paper the fields for the total rotation and the lateral displacement fields are coupled by using a coupling equation, which is derived for obtaining the static solution of shear deformable moderately thick square plates(C.M Wang et al 2005). This method reduces the magnitude of the problem by reducing the number of unknowns from $2 \mathrm{n}$ to $\mathrm{n}$, for the multi-term admissible functions and from two to one for the single term admissible functions and decreases the effort involved in the solution procedure considerably to obtain the fundamental frequency parameters.

The practical utility of the CDF method is demonstrated by solving the vibration problem of uniform and isotropic moderately thick square plates using the CDF method. The solution procedure and the numerical results obtained speak for themselves about the simplicity of the CDF method applied to the large amplitude free vibration problem of the moderately thick square plates compared to the finite element method. The numerical results for moderately thick plates, with all edges simply supported boundary condition are given along with the results obtained from the finite element method(K.K.Raju et al 1978) and the comparison clearly shows the efficacy of the proposed CDF method.

\section{First order shear deformation theory of plates}

The simplest shear deformation plate theory is the first order shear deformation plate theory (or FSDT), also referred to as the Mindlin plate theory (Mindlin, 1951) and it is based on the displacement field.

$$
\begin{aligned}
& u(x, y, z)=z \theta_{x}(x, y) \\
& v(x, y, z)=z \theta_{y}(x, y) \\
& w(x, y, z)=w(x, y)
\end{aligned}
$$

Where $\mathrm{u}$ and $\mathrm{v}$ are in plane displacements in $\mathrm{x}$ and $\mathrm{y}$ direction, $\mathrm{w}$ is transverse displacement along $\mathrm{z}$ direction, $\theta_{\mathrm{x}}$ and $\theta_{\mathrm{y}}$ denote rotations about the $\mathrm{y}$ and $\mathrm{x}$ axes respectively. In FSDT, shear correction factors are introduced to correct the discrepancy between the actual transverse shear stress distribution and that computed using the kinematic relations of FSDT. The shear correction factor $\mathrm{k}$ depend not only on the geometric parameters, but also on the loading and boundary conditions of the plate. However, a value of $\mathrm{k}=5 / 6$, the widely used value of the shear correction factor, is used in the present study.

\section{Coupled Displacement Field (CDF) method for plates}

In this method an admissible functions for $\theta_{\mathrm{x}}$ and $\theta_{\mathrm{y}}$ which satisfies all the geometric boundary conditions of plate domain are assumed. Note that these functions may satisfy some or all the natural boundary conditions also. The field for lateral displacement $\mathrm{w}$ is evaluated using the coupling equation taken from the static equilibrium equation independent of the externally applied load term(C.M Wang et al 2005).

$$
\frac{d w}{d x}=-\theta_{x}+\frac{h^{2}}{3.5}\left[\frac{\partial^{2} \theta_{x}}{\partial x^{2}}+v \frac{\partial^{2} \theta_{y}}{\partial y \partial x}\right]+\frac{h^{2}}{10}\left[\frac{\partial^{2} \theta_{x}}{\partial y^{2}}+\frac{\partial^{2} \theta_{y}}{\partial y \partial x}\right]
$$

It may be noted that only one coupling equation is obtained for the square plate. Though the admissible functions $\theta_{\mathrm{x}}$ and $\theta_{\mathrm{y}}$, in general can be written in a series form, here a single term admissible functions for $\theta_{\mathrm{x}}$ and $\theta_{\mathrm{y}}$ is chosen again with same intention of simplicity and better understanding of the CDF method as

$$
\begin{aligned}
& \theta_{x}=\alpha f_{1}(x, y) \\
& \theta_{y}=\alpha f_{1}(x, y)
\end{aligned}
$$

where $\alpha$ is the undetermined coefficient and $f_{1}(x)$ is the single term admissible function. Note that the functions for $\theta_{\mathrm{x}}$ and $\theta_{\mathrm{y}} \quad$ are the same, as the square plate is considered in the present study.

Substituting the admissible functions for $\theta_{x}$ and $\theta_{y}$ as given in (5) and (6) in (4), the coupled displacement field for the lateral displacement $\mathrm{w}$ is obtained, as

$w=\alpha f_{2}(x)$

Note that because of the use of the coupling equation, the transverse displacement distribution w contains the same undetermined coefficient $\propto$ as existing in the $\theta_{x} \& \theta_{y}$ distribution. In general, a $2 \mathrm{n}$ undetermined coefficients problem in the R-R method reduces to an $n$ undetermined coefficient problem in the CDF method. The linear fundamental frequency parameter is obtained from the following simple equation.

$\frac{d(U-T)}{d \alpha}=0$

The functions $f_{1}(x)$ and $f_{2}(x)$ are given later as per the boundary conditions of the plate considered

\section{Linear Vibrations}

The detailed procedure for the CDF method is discussed in this section for the case of evaluating the fundamental linear frequency parameter of a uniform simply supported moderately thick square plate for which the exact vibration mode shape (for shear flexible plate)for the transverse displacement $\mathrm{w}$ is well known. In the $\mathrm{CDF}$ method the admissible functions for $\theta_{x}$ and $\theta_{y}$ are assumed in the functional form, noting the similarity between $\frac{\partial w}{\partial x}, \theta_{x}$ and $\frac{\partial w}{\partial y}, \theta_{y}$ and satisfy the boundary conditions and symmetric conditions for the fundamental mode as

$$
\begin{aligned}
& \theta_{x}=\alpha \frac{\pi}{a} \cos \frac{\pi x}{a} \sin \frac{\pi y}{a} \\
& \theta_{y}=\alpha \frac{\pi}{a} \sin \frac{\pi x}{a} \cos \frac{\pi y}{a}
\end{aligned}
$$




$$
\frac{d w}{d x}=-\theta_{x}+\frac{h^{2}}{3.5}\left[\frac{\partial^{2} \theta_{x}}{\partial x^{2}}+v \frac{\partial^{2} \theta_{y}}{\partial y \partial x}\right]+\frac{h^{2}}{10}\left[\frac{\partial^{2} \theta_{x}}{\partial y^{2}}+\frac{\partial^{2} \theta_{y}}{\partial y \partial x}\right]
$$

substituting ( 7 ) and (8)in (9) and after integration and after evaluation of the constant of integration

$$
\begin{aligned}
& w=-\alpha \sin \frac{\pi x}{a} \sin \frac{\pi y}{a}\left[1+5.639 \oint\left(\frac{h}{a}\right)^{2}\right] \\
& U=\frac{D^{a}}{2} \int_{00}^{a a}\left\{\left(\frac{\partial \theta_{x}}{\partial x}\right)^{2}+\left(\frac{\partial \theta_{y}}{\partial y}\right)^{2}+2 v \frac{\partial \theta_{x}}{\partial x} \frac{\partial \theta_{y}}{\partial y}+2(1-v) \frac{\partial \theta_{x}}{\partial y} \frac{\partial \theta_{y}}{\partial x}\right\} d x d y+ \\
& \frac{k G h}{2} \int_{0}^{a} \int_{0}^{a}\left\{\left(\frac{d w}{d x}+\theta_{x}\right)^{2}+\left(\frac{d w}{d y}+\theta_{y}\right)^{2}\right\} d x d y
\end{aligned}
$$

Substituting (7), (8) and (10)in (11) and after simplification as

$$
U=\frac{D}{2} \alpha^{2}\left(\frac{\pi}{a}\right)^{4} a^{2}\left\{1+5.639\left\{\frac{h}{a}\right)^{2}\right\}
$$

The expression for kinetic energy of plate is given as $\mathrm{T}=\frac{\rho h w_{L}^{2}}{2} \int_{0}^{a} \int_{0}^{a}\left[w^{2}+\frac{h^{2}}{12}\left(\theta_{x}^{2}+\theta_{y}^{2}\right)\right] d x d y$

Substituting (7), (8) and (10) in the above (13) and after simplification

$\mathrm{T}=\frac{\rho h \omega_{L}^{2}}{2} \alpha^{2} \frac{a^{4}}{4}\left[\left(1+5.639 \oint\left(\frac{h}{a}\right)\right)+1.644 \oint\left(\frac{h}{a}\right)^{2}\right]$

By minimizing the Lagranzian with respect to undetermined coefficient $\alpha$

$$
\begin{aligned}
& \frac{\partial(U-T)}{\partial \alpha}=0 \\
& \lambda=\frac{\rho h \omega_{L}^{2} a^{4}}{D}=\frac{4 \pi^{4}\left(1+5.6398\left(\frac{h}{a}\right)^{2}\right)}{\left(\left[1+5.639\left(\frac{h}{a}\right)^{2}\right]+1.6499\left(\frac{h}{a}\right)^{2}\right)}
\end{aligned}
$$

\section{Large amplitude vibrations}

Large amplitude free vibrations can be studied, once the coupled displacement field for the lateral displacement $\mathrm{w}$ for an assumed $\theta_{x}$ and $\theta_{y}$ is evaluated using the principle of conservation of total energy at any instant of time which states that

$\mathrm{U}+\mathrm{T}+\mathrm{W}=$ Constant

The tension developed in the plate in $\mathrm{x}$-direction as

$T_{x}=\frac{E h}{2 a} \int_{0}^{a}\left\{\frac{d w(x)}{d x}\right\}^{2} d x$

$T_{x}=\frac{E h}{4} \alpha^{2} \frac{\pi^{2}}{a^{2}}$

Substituting $D=\frac{E h^{3}}{12\left(1-v^{2}\right)}$ in the above equation

$T_{x}=3\left(\frac{\alpha}{h}\right)^{2}\left(\frac{\pi}{a}\right)^{2} D\left(1-v^{2}\right)$

The tension developed in the plate does not contain any shear flexible terms.
$W=\frac{1}{2} T_{x} \int_{0}^{a} \int_{0}^{a}\left\{\frac{1}{2}\left(\frac{d w}{d x}\right)^{2}+\frac{1}{2}\left(\frac{d w}{d y}\right)^{2}\right\} d x d y$

Substituting (10) and (18) in (19) and after simplification

$W=\frac{3}{8} \pi^{4} D\left(1-v^{2}\right)\left[1+5.6398\left(\frac{h^{2}}{a}\right)\right]^{2}\left(\frac{\alpha}{h}\right)^{2} \frac{1}{a^{2}} \alpha^{2}$

Large amplitude free vibrations can be studied, once the coupled displacement field for the lateral displacement ' $w$ ' for an assumed $\theta_{z}$ and $\theta_{y}$ distribution is evaluated using the principle of conservation of total energy at any instant of time, neglecting damping, which states that

$\mathrm{U}+\mathrm{T}+\mathrm{W}=$ constant

Substituting (12), (14) and (20) in (21) and after simplification

$\dot{\bullet}^{2}+\alpha_{1} q^{2}+\alpha_{2} q^{4}=$ constant

where

$\alpha_{1}=\frac{\rho h \omega_{L}^{2} a^{4}}{D}=\frac{4 \pi^{4}\left(1+5.6398\left(\frac{h}{a}\right)^{2}\right)}{\left(\left[1+5.639 \oint\left(\frac{h}{a}\right)^{2}\right]^{2}+1.644 \oint\left(\frac{h}{a}\right)^{2}\right)}$

$\alpha_{2}=\frac{\rho h \omega_{N L}^{2} a^{4}}{D}=\frac{3\left(1-v^{2}\right) \pi^{4}\left[1+5.639 \oint\left(\frac{h}{a}\right)^{2}\right]^{2}\left(\frac{\alpha}{h}\right)^{2}}{\left\{\left[1+5.6398\left(\frac{h}{a}\right)^{2}\right]^{2}+1.644 \oint\left[\frac{h}{a}\right]^{2}\right\}}$

By following the harmonic balance method[3] the expression for frequency ratio as

$\left[\frac{\omega_{N L}}{\omega_{L}}\right]^{2}=1+1.5\left(\frac{\alpha_{2}}{\alpha_{1}}\right)\left(\frac{\alpha}{h}\right)^{2}$

$\left.\frac{\alpha_{2}}{\alpha_{1}}=\frac{3\left(1-v^{2}\right)}{4}\left[1+5.6398 \frac{h}{a}\right]^{2}\right]\left(\frac{\alpha}{h}\right)^{2}$

Substituting (25) in (24) and after simplification as

$\left[\frac{\omega_{N L}}{\omega_{L}}\right]=\sqrt{\left.1+1.0237 \$ 1+5.6398\left(\frac{h}{a}\right)^{2}\right]\left(\frac{\alpha}{h}\right)^{2}}$

From (26) frequency ratio is function of $\left(\frac{h}{a}\right)$ and amplitude ratio. In the case of a all edges clamped square plate the trigonometric admissible function for $\theta_{x}$ and $\theta_{y}$ that satisfies the required boundary conditions is taken as

$$
\begin{aligned}
& \theta_{x}=a \frac{2 \pi}{a} \sin \frac{2 \pi x}{a}\left[1-\frac{\cos 2 \pi y}{a}\right] \\
& \theta_{y}=a \frac{2 \pi}{a} \sin \frac{2 \pi y}{a}\left[1-\frac{\cos 2 \pi x}{a}\right]
\end{aligned}
$$

and by following the similar procedure as discussed for all edges simply supported square plate the ratios of the nonlinear to the linear radian frequencies can be obtained for the clamped-clamped square plate as 


$$
\left(\frac{\omega_{N L}}{\omega_{L}}\right)^{2}=1+0.2559 \frac{\left[1.5+381.6475\left(\frac{h}{a}\right)^{4}+45.116\left(\frac{h}{a}\right)^{2}\right]}{\left[1+16.917 f\left(\frac{h}{a}\right)^{2}\right]}
$$

\section{Numerical results and discussion}

Using the formulation described above, the large amplitude vibration behavior of a uniform moderately thick square plate is obtained in terms of $\frac{\omega_{N L}}{\omega_{L}}$ or $\frac{T_{N L}}{T_{L}}$ (Ratio of non-linear radian frequency to the linear radian frequency or non-linear time period to the linear time period) in terms of various $\frac{\alpha_{m}}{h}$ (maximum amplitude ratios) and $\mathrm{h} / \mathrm{a}$ (thickness ratios). As a demonstration of the proposed formulation, the plate is considered with axially immovable edges (Fig.1).

The present results in terms of $\frac{T_{N L}}{T_{L}}$ are presented in Table1for all edges simply supported and Table 2 for all edges clamped. For the sake of comparison and validation of the proposed method, the same results obtained by the finite element method FEM(K.K.Raju et al 1978 ) are included in Tables 1 and 2. The present results for the extreme case of $\mathrm{h} / \mathrm{a}=0.001$ and $\mathrm{h} / \mathrm{a}=0.2$ match very well with the results of FEM, the maximum error being $1.5 \%$ of this extreme case.

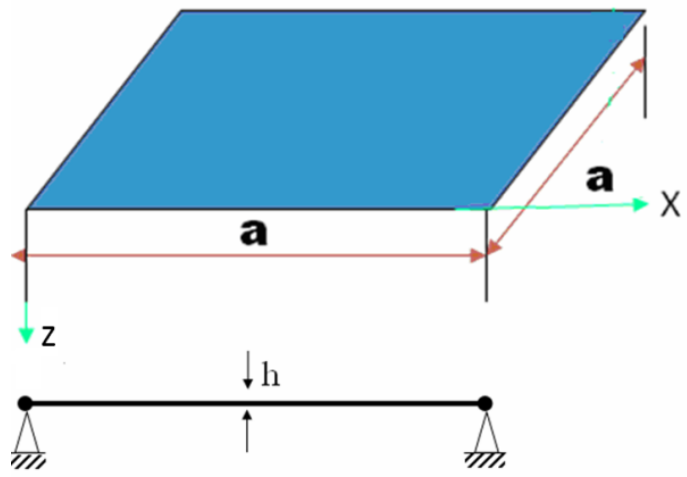

Fig.1 Uniform all edges simply supported moderately thick square plate

\section{Conclusions}

The concept of coupled displacement field, applicable to square plates, presented in this paper is successfully applied to study the large amplitude free vibration behavior of uniform shear flexible plate with edges immovable. Elegant and accurate closed form expressions for $\frac{\omega_{N L}}{\omega_{L}}$ for all edges simply supported and clamped are obtained in terms of thickness ratios and maximum amplitude ratios. The results obtained using the present formulation are found to be in good agreement with those results obtained by FEM for a wide range of maximum amplitude ratios, including the thin plates and moderately thick plates.

\section{Acknowledgments}

The first author is highly thankful to authorities of University College of Engineering(A), JNTU-Kakinada for sponsoring the necessary economical support for presenting the paper under TEQIP2. Second author gratefully acknowledges the support given by Mallareddy college of engineering during the course of this work.

\section{References}

D Zhou, (2001) Free vibration of multi-span Timoshenko beams using static Timoshenko beam functions, Journal of Sound and Vibration, 241, 725-734.

R. D. Mindlin,(1951) Influence of rotary inertia and shear on flexural motions of isotropic, elastic plates, Journal of Applied Mechanics, 18, 31-38.

G.Venkateswara Rao and K.Meera Saheb,(2004) Large amplitude free vibrations of shear flexible uniform beams through coupled displacement field, VETOMAC-3, 2, 1167 1174.

G.Venkateswara Rao and K.Meera Saheb,(2005) Large amplitude free vibrations of shear flexible uniform beams through coupled displacement field concept, Advances in Vibration Engineering, 4(3), 213-220

K.Meera Saheb, G.Venkateswara Rao and G.Rangajanardhan (2005)Evaluation of large amplitude free vibration behavior of uniform Timoshenko beams using Coupled Displacement Field method,VETOMAC-4

G.Venkateswara Rao, K.Meera Saheb and G. Rangajanardhan, (2006)Fundamental frequency for large amplitude vibrations of uniform Timoshenko beams with central point concentrated mass using coupled displacement field method, Journal of Sound and Vibration, 298,221-232G.Venkateswara Rao, K.Meera Saheb and

G. Rangajanardhan, (2006)Concept of coupled displacement filed for large amplitude free Vibrations of shear flexible beams, Journal of Vibration and Acoustics, 128, 251-255

K.Meera Saheb, G.Venkateswara Rao and G.Ranga Janardhana,(2007) Free vibration analysis of Timoshenko beams using coupled Displacement Field Method, Journal of Structural Engineering, 34(3), 233-23

K.Meera Saheb, G.Venkateswara Rao and G.Ranga Janardhana, (2009)Buckling and free vibration of moderately thick square plates using coupled displacement field method, Journal of Structural Engineering, 36(4), 155-159.

K.Meera Saheb, G.Venkateswara Rao and G.Rangajanardhan(2010) Evaluation of large amplitude free vibration behavior of uniform Timoshenko beams using Coupled Displacement Field method, Advances in Vibration Engineering, 9 (1), 15-23.

K.K.Raju, G.V.Rao, and I.S. Raju, (1978)Effect of geometric nonlinearity on large amplitude free flexural vibrations of moderately thick rectangular plates, Computers and Structures, $9,441-444$

Wah, T., 1963, Large Amplitude Flexural Vibration of Rectangular Plates, International. Journal of Mechanical Sciences., 5, pp.425-438.

Chu, H.N., and Herrman, G., 1956, Influence of Large Amplitudes on Free Flexural Vibrations of Rectangular Elastic Plates, Journal of Applied Mechanics, 23, pp.532-540

Chu Mei, 1973, Finite Element Displacement method for Large Amplitude Free Flexural Vibrations of Beams and Plates, Computers \& Structures, 3, pp.163-17

Wang,C.M. Wang,C.Y.and Reddy,J.N..,(2005) Exact solutions for buckling of structural members, CRC Press. 